\title{
CORRESPONDENCE
}

\section{IPCC yet to assess geoengineering}

As co-chair of Working Group III of the Intergovernmental Panel on Climate Change (IPCC), I would like to clarify your misleading implication that I recommend geoengineering as an option for attaining ambitious climate targets (Nature 468, 13-14; 2010).

The IPCC does not have a mandate to recommend or dismiss specific policies or technologies. It aims to assess all relevant climate-change mitigation options on the basis of peer-reviewed scientific literature. This assessment must be unbiased, factual and policy-relevant, without being prescriptive.

There is ongoing public and scientific debate about using geoengineering as one of several options to mitigate climate change. Some decision-makers consider it a last resort in case technologies that are more conventional prove insufficient (for example, usage of renewable energy, carbon dioxide capture and storage, or nuclear power). Others are against a geoengineering option because of gaps in our knowledge, unwanted side effects and unmanageable risks. Notably, delegates at the United Nations Convention on Biological Diversity summit last month in Nagoya, Japan, agreed to call for a moratorium on geoengineering schemes (see go.nature.com/ kc1b9c). Also, the governance structure of this option needs further consideration. Several fundamental questions must be answered before the deliberate alteration of Earth's radiation balance can be considered as a reliable option.

The IPCC will therefore assess the risks, feasibility, mitigation potential, costs and governance requirements of geoengineering for the first time in its Fifth
Assessment Report, due to be released in 2013-14.

Meanwhile, the three IPCC working groups are coordinating a meeting of expert scientists from different disciplines as a platform for exchange and discussion. The aim is to encourage research into the implications of different geoengineering technologies, as well as to study their potential impact on biodiversity, human society and the climate system. Ottmar Edenhofer Potsdam Institute for Climate Impact Research, Germany. ottmar.edenhofer@ipcc-wg3.de

\section{An intellectual black hole}

News items on climate change are now regularly flooded with negative online comments (see, for example, go.nature.com/ Lfigec). These tend to have certain features in common.

The comment writers struggle to find words that are emphatic enough to express their contempt for anthropogenic global warming and for the $97 \%$ or so of active climate scientists who accept its reality. They feel attacked, lied to and conspired against: they are intensely angry. Their comments are often pervaded by heavy sarcasm. In their view, climate scientists are not merely mistaken, but foes of truth and liberty. They see themselves as fighting a powerful enemy - a posture that can be addictive.

These comment authors rarely engage with the original science (in the above example, an article in Geophysical Research Letters). They have unplugged from the scientific discourse because they believe it to be driven by crypto-environmentalist (or crypto-communist) ideology. This conviction characterizes fundamentalisms: a true believer does not need to engage, they already know. The denialists charge that it is the scientists who refuse to consider the evidence; alternatively, scientists know all about it and are lying. The parallels with some creationist rhetoric are striking.

Certain standard fallacies and counterfactuals are held by these commenters as irresistible 'gotchas' - any one of which makes the idea of humaninduced global warming absurd (and further thought unnecessary). For example, a popular line of argument is that because climate has changed in the past without human input, humans cannot be changing it now. Mistaken beliefs are held that scientists on the Intergovernmental Panel on Climate Change, climate modellers and other researchers ignore factors such as water vapour, urban heat islands and the Sun.

The peculiar danger of any full-blown conspiracy theory is that it can become an intellectual black hole, a oneway trip. Hope lies mostly in keeping people out of the hole, rather than trying to rescue those who have fallen in. Larry Gilman Sharon, Vermont, USA.lgilman@myfairpoint.net

\section{Space telescope is worth the effort}

Your sensationalist headline 'The telescope that ate astronomy' could more appropriately have highlighted the promise of NASA's James Webb Space Telescope (JWST) for astronomy's future (Nature 467, 1028-1030; 2010).

No facilities exist on or off this planet - or are called for in the recent US decadal survey of astronomy and astrophysics - for finding the first galaxies or for detecting liquid water on habitable planets around other stars. These capabilities have been part of the JWST science plan since its inception.

Such transformative science is why NASA, the European Space Agency and the Canadian Space Agency took on the challenge of building a telescope of comparable scale to the ground-based W. M. Keck telescopes or one of Europe's Very Large Telescopes. This meant reducing the JWST's mass by almost two orders of magnitude compared with a Keck telescope, cooling it to $-313^{\circ} \mathrm{C}$ and folding it to fit inside an Ariane 5 spacecraft for deployment 1.5 million kilometres from Earth.

Building the JWST has required the manufacture of 18 1.3-metre beryllium mirrors able to hold their shapes to better than 20 nanometres at cryogenic temperatures, and of a deployable gossamer sunshield the size of a tennis court.

It was equally challenging to build the Hubble Space Telescope. But it was worth it: Hubble has revolutionized our understanding of the Universe, inspired generations of school children with its breathtaking images and supported a community of 8,000 astronomers worldwide. Likewise, the efforts of today's space scientists are creating a revolutionary telescope for the next generation of scientists and engineers.

Matt Mountain, John Grunsfeld Space Telescope Science Institute, USA.mmountain@stsci.edu Heidi Hammel Space Science Institute, USA.

\section{CONTRIBUTIONS}

Readers are welcome to comment online on anything published in Nature: www.nature.com/ nature. Submissions to Correspondence may be sent to correspondence@ nature.com. 